\title{
Winding branes and persistent currents
}

\author{
Sergei Khlebnikov* \\ Department of Physics, Purdue University, West Lafayette, IN 47907
}

\begin{abstract}
A D5 brane winding around a stack of D3 branes can be used as a model of persistent current in a thin superconducting ring, with the number $N$ of D3s corresponding to the number of transverse channels in the ring. We consider, in the large $N$ limit, existence and properties of a gapped superconducting state with a uniform winding number density $q$. We find that there is a gapped classical solution for any $q$, no matter how large, but when $q$ is larger than a certain $q_{m}$ the state is unstable with respect to decay by phase slips. We argue that this decay produces strings via a version of the Hanany-Witten effect (in a non-transverse, non-supersymmetric arrangement of branes). This parallels the requirement of quasiparticle production in a clean (disorder-free) wire in field theory of superconductivity.
\end{abstract}

${ }^{*}$ skhleb@physics.purdue.edu 


\section{Contents}

1 Introduction

2 A review of the BdG formalism

3 Computation of the WZ term 7

4 Numerical solutions $\quad 10$

5 Worldvolume flux and charge 11

6 Computation of free energies 13

7 Discussion 17

\section{Introduction}

In this paper, we consider solutions of type IIB supergravity that describe one brane winding around another. The specific example we choose corresponds to the configuration in string theory that consists of a stack of $N$ D3 branes and a single D5 brane, in the following arrangement:

$$
\begin{array}{ccccccccccc} 
& 0 & 1 & 2 & 3 & 4 & 5 & 6 & 7 & 8 & 9 \\
N \text { D3s: } & \times & \times & \times & \times & & & & & & \\
\text { D5: } & \times & \times & & & \times & \times & \times & \times & &
\end{array}
$$

where crosses denote the coordinates wrapped by the branes. The only spatial direction shared by all the branes is $x \equiv x^{1}$, which we assume to be a circle, possibly of a large radius. We will be interested in the case when, as one goes around the circle, the D5 winds around the D3s, the total of $W$ times.

Our interest in this configuration is that we believe it to be suitable for description of a current-carrying state in a thin superconducting ring, with $W$ corresponding to the winding number of the order parameter. There are questions concerning thin superconductors, such as, for instance, conditions of quantum stability of the supercurrent, that are not easily answered by the conventional mean-field theory, and we would like to see if supergravity can help us to answer these questions.

At small values of the 't Hooft coupling $\lambda=g_{s} N$ (where $g_{s}$ is the closed string coupling), one may start by considering the trivial embedding - with the D3s at $x^{4}=$ $\ldots=x^{9}=0$, and the D5 at $x^{2}=x^{3}=x^{8}=x^{9}=0$-and developing a field theory around it. This corresponds to replacing strings connecting the D5 to the D3s with 
their ground states and results in a non-supersymmetric gauge theory of $N$ species of left- and right-moving massless defect fermions. These correspond to $N$ channels of conduction electrons in a wire $\dagger^{\dagger}$

The role of interactions, on the other hand, is most readily understood in the large $N$ limit, when $g_{s}$ is small but $\lambda$ is large. In this case, it is possible to replace the D3 branes by the classical black brane carrying $N$ units of the D-brane charge [1, i.e., by one of the solutions found in [2], while the D5 can be considered as a probe. The near-horizon region of the D3 geometry has a field-theoretical interpretation even at strong coupling: it is dual to an $S U(N)$ superconformal gauge theory [3, 4, 5]. In our case, however, the D5 will not stay in that region: once displaced from the trivial embedding, it is pushed out to large absolute values of

$$
x^{8}+i x^{9}=\Delta e^{i \phi} .
$$

The correspondence of [3, 4, 5] is not applicable at large $\Delta$, so supergravity is the only available microscopic description of this strongly coupled system. It will be our starting point in what follows.

We interpret the instability of the trivial embedding as formation of a superconducting state [6]. The latter is represented by a nontrivial embedding, which we find numerically for various values of the winding of the phase $\phi$. A nonzero winding corresponds to a nonzero supercurrent in the wire. All embedding solutions we consider here are uniform in the $x$ direction and so describe a superconductor in the clean limit (i.e., without disorder). In particular, the gradient of $\phi$ (the winding number density, in units of $1 / 2 \pi)$ is independent of $x$ and given by

$$
q=\frac{2 \pi W}{L}
$$

where $L$ is the length of the wire. In practice, disorder is often important, and the first step towards including it in our description would be to consider $x$-dependent background geometries. We discuss this further in the concluding section.

We find that there is a nontrivial embedding solution for any value of $q$, no matter how large. For each of these solutions, the minimal distance from the D5 to the D3sthe quasiparticle gap in units of the string tension - is nonzero. This is in contrast to the case of fixed current (and zero winding) we have considered in [6]. In that case, the superconducting solution is gapless at any nonzero current and disappears at a

\footnotetext{
$\dagger$ In a wire, different channels correspond to different transverse wavefunctions and different projections of spin. Thus, at large $N$, the material we are describing is one-dimensional only in its superconducting properties (e.g., the value of the order parameter), while the electrons are free to move in all three directions.
} 
critical current. The distinction between the fixed-winding and fixed-current cases has a parallel in the conventional mean-field theory, as we discuss in Sec. 2 ,

The existence of a gapped classical solution for any $q$ may seem surprising: the prevalent situation in superconductivity is when, at a certain $q=q_{L}$, quasiparticles with momenta antiparallel to the flow become gapless, and the supercurrent becomes classically unstable. This stability bound is known as Landau's criterion; we recall its derivation in Sec. 2. Note, however, that our superconductor is perfectly uniform and therefore momentum-conserving, while the Landau process is not. So, it is possible that an instability of this type will not show up until we break momentum conservation by going over to an $x$-dependent background.

Next, we consider stability of the supercurrent with respect to phase slips. These are events (either thermal [7] or quantum [8]) that remove a unit of winding from the supercurrent. Since reducing supercurrent releases momentum from it, a sink of momentum is required. Unlike in the Landau process, however, that sink may be a part of the system itself, so that no momentum needs to be supplied from the outside (e.g., by disorder). The existing microscopic theory of this effect [9] is based on the Bogoliubov-de Gennes (BdG) equations, which, when interpreted liberally enough (as reviewed in Sec. 2), allow one to go one step beyond the conventional mean field, to include fluctuations of the order parameter. The result is that, in a uniform system at zero temperature, a phase slip must produce a certain number of fermionic quasiparticles (with total momentum parallel to the flow), and the associated energy cost may block the process altogether.

The liberal interpretation of the $\mathrm{BdG}$ equations, referred to above, treats the order parameter and the quasiparticles as separate quantum fields. One may be concerned that this involves double counting, as both are ultimately made of the same electrons. One may counter this by arguing that, as in any two-fluid model, the two fields simply represent two different transport mechanisms (in our case, of the electric charge), and the separation of the electrons into superconducting and normal should not be construed too literally. Nevertheless, it is of interest to develop an approach in which the superconducting and normal components are represented by different types of excitations - e.g., an elementary field vs. a soliton or a string. Dual gravity provides precisely this type of approach.

An important role in our calculation is played by the Wess-Zumino (WZ) term in the D5 action. We find that for any nonzero winding $W$ it causes the D5 to have an amount, equal to $N W$, of the charge conjugate to the worldvolume gauge field. Since a QPS changes $W$, it changes the amount of charge and, by charge conservation, the difference has to be picked up by excitations. We interpret this transfer of charge as a version of the Hanany-Witten (HW) effect [10]: when a D5 brane crosses a D3, a 
fundamental string is produced. This version of the effect is similar but not identical to the one well known in the literature, in which the D3 and D5 have no common spatial directions and preserve some supersymmetry [11, 12, 13. We find it remarkable that the present version completely parallels the requirement of quasiparticle production obtained in field theory of superconductivity on the basis of the BdG equations.

While a phase slip reduces the energy of the supercurrent, to see if it is in fact energetically allowed, we must take into account the energy of the produced quasiparticles. We find that single phase slips are energetically forbidden until the winding number density reaches a certain $q_{m}$. Thus, although a solution with nonzero supercurrent exists for any $q$, at $q>q_{m}$ it is unstable with respect to decay by phase slips. We discuss some consequences of this in the concluding section.

\section{A review of the $\mathrm{BdG}$ formalism}

The BdG formalism is not used for any calculations in this paper. We review it here as a reference point for later comparison with the results obtained using gravity and also to illustrate the distinction between the fixed-winding and fixed-current cases.

Consider the following Lagrangian, describing $N$ species of $(1+1)$ dimensional fermions, $\psi_{n}$, which interact with an order parameter field $\Phi$ :

$$
L_{F}=\sum_{n}\left(i \bar{\psi}_{n} \gamma^{\alpha} \partial_{\alpha} \psi_{n}-\frac{1}{2} \Phi \bar{\psi}_{n} \psi_{n}^{c}-\frac{1}{2} \Phi^{*} \bar{\psi}_{n}^{c} \psi_{n}+\mu \bar{\psi}_{n} \gamma^{0} \psi_{n}\right)
$$

Here $\alpha=0,1$ and $n=1, \ldots, N$. The superscript $c$ denotes charge conjugation, and $\mu$ is a chemical potential. Each $\psi_{n}$ is a two-component Dirac spinor. We use the representation of the $\gamma$ matrices with $\gamma^{0}=\sigma_{1}, \gamma^{1}=-i \sigma_{2}$, where $\sigma$ s are the Pauli matrices. Thus, in the normal state $(\Phi=0)$, the upper components of $\psi_{n}$ describe right-, and the lower left-, moving fermions.

The Dirac fermions $\psi_{n}$ can be related to the electron operators $a_{R, L}$ of a superconductor by taking $N$ even and setting, for each $\nu=1, \ldots, N / 2$,

$$
\psi_{2 \nu-1}=\left(\begin{array}{c}
a_{R \nu \uparrow} \\
a_{L \nu \downarrow}
\end{array}\right), \quad \psi_{2 \nu}=\left(\begin{array}{c}
a_{R \nu \downarrow} \\
-a_{L \nu \uparrow}
\end{array}\right),
$$

where an arrow denotes the projection of spin. The minus sign in the second instance in (5) causes $\Phi$ to couple to a spin singlet, the case for most conventional superconductors. The index $\nu$ labels the transverse channel. It reflects the fact that, while the fields $\psi_{n}$ are functions of time and $x$ only, the electrons in a superconductor

move in all three spatial directions and can be classified according to their transverse wavefunctions. 
The BdG equations are obtained from (44) by variation with respect to $\psi_{n}$. Our interpretation of these equations will be broader than the conventional one: in most applications $\Phi$ is considered as a classical background, but we will allow quantum changes in $\Phi$. This is necessary if we wish to use (4) to describe quantum phase slips.

In mean-field theory, one can describe a steady supercurrent as a state with a uniformly wound order parameter:

$$
\Phi(x)=\Phi_{0} e^{i q x}
$$

where $\Phi_{0} \neq 0$ is a constant. A quantum phase slip is described by a configuration (instanton) that interpolates in the Euclidean time between the state (6) and a similar state with a smaller $q$, corresponding to one fewer unit of winding. Such an instanton has one zero mode for each fermionic species [14, 15]. An adaptation of the argument of [16] then shows that $N$ fermions must be produced in the phase slip process [9].

The quasiparticle spectrum near the ground state ([6) can be conveniently obtained by redefining the Fermi fields as follows:

$$
\psi_{n}(x . t) \rightarrow e^{\frac{i}{2} q x} \psi_{n}(x, t)
$$

This unwinds $\Phi$ into a constant, $\Phi(x) \rightarrow \Phi_{0}$, but also modifies the Lagrangian:

$$
L_{F} \rightarrow L_{F}-\frac{q}{2} \sum_{n} \psi_{n}^{\dagger} \sigma_{3} \psi_{n}
$$

The additional term in (8) gives different chemical potentials to the right- and leftmoving fermions. The corresponding Hamiltonian can be diagonalized by a Bogoliubov transformation. The resulting excitation energies are

$$
\epsilon_{ \pm}(k)=\left[(k-\mu)^{2}+\left|\Phi_{0}\right|^{2}\right]^{1 / 2} \pm \frac{q}{2} .
$$

The upper branch, $\epsilon_{+}(k)$, describes excitations with momentum $k$, and the lower branch, $\epsilon_{-}(k)$, those with momentum $-k$. Both reach minima at $k=\mu$, which can therefore be identified with the Fermi momentum: $\mu=k_{F}$. The lower branch touches zero when $q$ becomes equal to

$$
q_{L}=2\left|\Phi_{0}\right|
$$

that is twice the quasiparticle gap. Once $q$ exceeds $q_{L}$, it becomes energetically favorable to produce excitations with momenta near $-k_{F}$, and the ground state becomes unstable. The stability condition $q<q_{L}$ is known as Landau's criterion. As we have noted in the introduction (and discuss further in the conclusion), we do not recover this condition in the gravity-based calculation, presumably because of the perfectly momentum-conserving nature of our system. 
We can now define two limiting types of current-carrying states. In one type, quasiparticles are absent but the order parameter is wound as in (6); the current this state carries is a supercurrent. We refer to this case as fixed winding. In the other type of state, $\Phi(x)=\Phi_{0}$, but the upper branch of the excitation spectrum (9) is filled with quasiparticles, up to a certain finite density of them. The current these carry is a normal current. Because the quasiparticles form a Fermi surface this state is gapless. We identify it as a mean-field counterpart of the gapless superconductor described via a gravity dual in [6]. We refer to this case as fixed current.

In general, one must allow for both supercurrent and normal current components to be present. As long as phase slips are neglected, the momenta of these components are separate conserved quantities. Phase slips, however, convert winding of the order parameter into momentum of quasiparticles and vice versa. The equilibrium winding and density of quasiparticles are then determined by comparing the free energies of different states with the same total momentum. In this paper, we will be interested in a condition under which phase slips become energetically favorable and the decay of the supercurrent-only state begins to populate the normal component.

\section{Computation of the $\mathrm{WZ}$ term}

Equations of motion for the D3 geometry can be derived from the action

$$
S_{10}=\frac{1}{2 \kappa^{2}} \int d^{10} x \sqrt{-g}\left(\mathcal{R}-\frac{1}{4 \times 5 !} G_{(5)}^{2}\right) .
$$

Here $\mathcal{R}$ is the Ricci scalar, and $G_{(5)}$ is the Ramond-Ramond 5-form field strength. We use the shorthand notation

$$
G_{(5)}^{2}=G_{A B C D E} G^{A B C D E}
$$

$G_{(5)}$ is self-dual, which implies $G_{(5)}^{2}=0$ but, as is common practice, we impose self-duality in the equations of motion, rather than directly in the action.

In the classical limit, a stack of $N$ extremal D3 branes is described by a solution [2] to the equations of motion following from (11), with

$$
d s^{2}=\frac{1}{\sqrt{f}}\left(-d t^{2}+\left(d x^{i}\right)^{2}\right)+\sqrt{f}\left(d r^{2}+r^{2} d \Omega_{5}^{2}\right),
$$

$(i=1,2,3)$ and

$$
G_{(5)}=Q\left(\epsilon_{(5)}+* \epsilon_{(5)}\right),
$$

\footnotetext{
${ }^{\ddagger}$ Not to be confused with the original electron Fermi surface at $k=\mu$.
} 
where $d \Omega_{5}^{2}$ is the metric on the unit 5 -sphere, and $\epsilon_{(5)}$ is the volume 5 -form on it. The metric function in (13) is

$$
f=1+\frac{R^{4}}{r^{4}}
$$

and the relation between the various parameters is

$$
Q=16 \pi g_{s}\left(\alpha^{\prime}\right)^{2} N=4 R^{4},
$$

where $g_{s}$ is the closed string coupling. The D3s themselves are hidden behind the degenerate horizon of (13) at $r=0$.

Note that we are using the full D3 geometry, rather than the near-horizon limit; the latter would correspond to neglecting unity in comparison with $R^{4} / r^{4}$ in (15). The physical significance of this has been discussed in the introduction. Technically, we need the full geometry because a probe D 5 placed in it will be repelled to large values of the radius $r, r_{\min } \sim R$.

The probe D5 is governed by the action [17]

$$
S_{D 5}=-\tau_{5} \int d^{6} \xi \sqrt{-\operatorname{det}\left(P[g]_{a b}+F_{a b}\right)}+\tau_{5} \int A \wedge P\left[G_{(5)}\right],
$$

where $\tau_{5}$ is the brane tension, $\xi^{a}$ with $a=0, \ldots, 5$ are coordinates on the brane, $P$ denotes pullbacks to the brane worldvolume, $A_{a}$ is the worldvolume gauge field, and

$$
F_{a b}=\partial_{a} A_{b}-\partial_{b} A_{a}
$$

is its field strength. The WZ term is the second term in (17).

In our case, the D5 wraps only three of the five angles appearing in (13), so it is convenient to rewrite the metric as

$$
d s^{2}=\frac{1}{\sqrt{f}}\left(-d t^{2}+\left(d x^{i}\right)^{2}\right)+\sqrt{f}\left(d \rho^{2}+\rho^{2} d \Omega_{3}^{2}+d \Delta^{2}+\Delta^{2} d \phi^{2}\right),
$$

where $\Delta$ and $\phi$ are polar coordinates in the $\left(x^{8}, x^{9}\right)$ plane. Then,

$$
r^{2}=\rho^{2}+\Delta^{2}
$$

In our calculation, the instability with respect to the D5 slipping off the equator of the $S^{5}$, i.e., developing a non-zero expectation value of $\Delta$, corresponds to a pairing instability towards a superconducting state.

With these notations, the coordinates on the brane are

$$
\xi^{a}=(t, x, \rho, \boldsymbol{\alpha})
$$

where $x \equiv x^{1}$, and $\boldsymbol{\alpha}=\left(\alpha^{1}, \alpha^{2}, \alpha^{3}\right)$ are the three angles spanning the $S^{3}$ in (19)). If we restrict attention to embeddings with $x^{2}=x^{3}=0$, the general form of the embedding 
is $\Delta=\Delta\left(\xi^{a}\right), \phi=\phi\left(\xi^{a}\right), A_{a}=A_{a}\left(\xi^{b}\right)$. It is, however, consistent to restrict the class of embeddings further, to

$$
\begin{aligned}
\Delta & =\Delta(\rho), \\
\phi & =q x, \\
A_{t} & =A_{t}(\rho),
\end{aligned}
$$

where $q$ is a constant, and all $A_{a}$ with $a \neq t$ are equal to zero.

For this class of embeddings, the WZ term in (17) becomes

$$
S_{W Z}=\tau_{5} \int d t d x d \rho d^{3} \alpha A_{t} P\left[G_{(5)}\right]_{x \rho \boldsymbol{\alpha}} .
$$

Only the magnetic part of (14) contributes to (25). The requisite components of $G_{(5)}$ are

$$
\begin{aligned}
G_{\Delta \phi \boldsymbol{\alpha}}(\rho, \boldsymbol{\alpha}, \Delta) & =-\Delta \rho^{3} \partial_{\rho} f(\rho, \Delta) \sqrt{g_{3}(\boldsymbol{\alpha})} \\
G_{\rho \phi \boldsymbol{\alpha}}(\rho, \boldsymbol{\alpha}, \Delta) & =\Delta \rho^{3} \partial_{\Delta} f(\rho, \Delta) \sqrt{g_{3}(\boldsymbol{\alpha})} \\
G_{\rho \Delta \boldsymbol{\alpha}}(\rho, \boldsymbol{\alpha}, \Delta) & =0
\end{aligned}
$$

where $g_{3}$ is the determinant of the metric on $S^{3}$ and

$$
f(\rho, \Delta)=1+\frac{R^{4}}{\left(\rho^{2}+\Delta^{2}\right)^{2}}
$$

[cf. (15) and (20)]. The requisite component of the pullback is

$$
P\left[G_{(5)}\right]_{x \rho \boldsymbol{\alpha}}=-q\left(G_{\Delta \phi \boldsymbol{\alpha}} \Delta_{, \rho}+G_{\rho \phi \boldsymbol{\alpha}}\right),
$$

where we use the shorthand $\Delta_{, \rho} \equiv \partial_{\rho} \Delta$. Substituting this into (25) and integrating over $\boldsymbol{\alpha}$, we obtain

$$
S_{W Z}=2 \pi^{2} q \tau_{5} R^{4} \int d t d x d \rho A_{t}(\rho) \frac{d}{d \rho} \Pi(\rho, \Delta(\rho)),
$$

where

$$
\Pi(\rho, \Delta)=\frac{\rho^{4}}{\left(\rho^{2}+\Delta^{2}\right)^{2}} .
$$

The full D5 action (17) for embeddings of the form (22)-(24) is

$$
S_{D 5}=-2 \pi^{2} \tau_{5} \int d t d x d \rho \sqrt{Z}\left(1+\Delta_{, \rho}^{2}-F_{t \rho}^{2}\right)^{1 / 2}+S_{W Z}
$$

where

$$
Z(\rho, \Delta)=\rho^{6} f(\rho, \Delta)\left[1+q^{2} \Delta^{2} f(\rho, \Delta)\right],
$$

with $f$ given by (29), and $\Delta$ by $\Delta(\rho)$. 


\section{Numerical solutions}

We call an embedding solution superconducting if it is nontrivial (i.e., $\Delta(\rho)$ is not identically zero) and, in addition,

$$
\Delta(\rho \rightarrow \infty)=0
$$

The latter condition means that that there is no superconductivity in the ultraviolet $\$$ If the D5 brane does not cross the horizon, i.e.,

$$
\Delta(\rho=0) \neq 0
$$

the minimal distance from it to the D3s is finite and so then is the quasiparticle gap. We refer to such solutions as gapped. As we will see, there is a gapped superconducting solution for any value of $q$. This is in contrast to the same system at fixed current [6], where the superconducting solution at any nonzero current is gapless 9

The case $q=0$ is 2-dimensionally Lorentz invariant and so is subject to Coleman's theorem [18] on nonexistence of Goldstone bosons in 2 dimensions. This implies that the long-range order (LRO) represented by the nontrivial profile of $\Delta$ is destroyed by quantum fluctuations. One may suspect that the same is true also for the Lorentz noninvariant case $q \neq 0 \|$ The strength of quantum fluctuations, however, is suppressed by the large $N$ (essentially, by the D5's tension in the gravity description or by the thickness of the wire). As a result, as $N$ increases, significant deviations from classical behavior occur at progressively larger spatial scales. Thus, our classical solutions faithfully represent the physics except at these largest scales. We also recall that superconductivity does not require an LRO; it only requires that the superconducting density does not renormalize to zero at large distances.

The equation of motion obtained from (33) by variation with respect to $A_{t}$ integrates into

$$
\frac{\sqrt{Z} F_{t \rho}}{\left(1+\Delta_{, \rho}^{2}-F_{t \rho}^{2}\right)^{1 / 2}}+q R^{4} \Pi=-J_{0},
$$

where $\Pi$ is given by (32) , and $J_{0}$ is the integration constant. Solving this algebraically for $F_{t \rho}$, we obtain

$$
F_{t \rho}=-\frac{J}{\sqrt{C}}\left(1+\Delta_{, \rho}^{2}\right)^{1 / 2}
$$

\footnotetext{
$\S$ In that respect, perhaps a better, if a bit cumbersome, name for these embeddings would be spontaneously superconducting.

IThe solution at zero current is gapped and coincides with the $q=0$ solution found here.

॥ In a gapless Lorentz non-invariant superconductor, the low-energy fluctuations of the order parameter can be damped by gapless quasiparticles. We assume that a finite gap prevents this mechanism of stabilizing an LRO from operating in the present case.
} 
where

$$
\begin{aligned}
J(\rho, \Delta) & =q R^{4} \Pi(\rho, \Delta)+J_{0}, \\
C(\rho, \Delta) & =Z(\rho, \Delta)+J^{2}(\rho, \Delta) .
\end{aligned}
$$

The condition (36) requires that $J_{0}=0$ : unless that is so, $F_{t \rho}(\rho=0) \neq 0$, and $A_{t}$ cannot be smooth at $\rho=0$.

Substituting (38) into the equation obtained by variation of (33) with respect to $\Delta$, we obtain

$$
\frac{d}{d \rho} \frac{\sqrt{C} \Delta_{, \rho}}{\left(1+\Delta_{, \rho}^{2}\right)^{1 / 2}}=\left(1+\Delta_{, \rho}^{2}\right)^{1 / 2} \frac{\partial \sqrt{C}}{\partial \Delta},
$$

where $C$ is given by (40) (and $J$ by (39), with $J_{0}=0$ ). Regularity of $\Delta(\rho)$ at $\rho=0$ together with (36) leads to the boundary condition

$$
\Delta_{, \rho}(\rho=0)=0
$$

Thus, (41) has to be solved with the boundary conditions (35) and (42). We do that numerically by shooting from $\rho=0$ with $\Delta(\rho=0)$ as a shooting parameter.

Some representative solutions are shown in Fig. 1, We find that such a solution exists for any $q$, no matter how large. The solution approaches a fixed shape as $q \rightarrow \infty$. The absence of a classical stability bound on $q$ (i.e., of the Landau criterion) has been remarked upon in the introduction and is further discussed in the conclusion. The minimal value of

$$
r(\rho)=\left[\rho^{2}+\Delta^{2}(\rho)\right]^{1 / 2},
$$

which gives the quasiparticle gap in units of the string tension, is reached at $\rho=0$. Thus, the gap is given by $\Delta(\rho=0)$. A somewhat counterintuitive result is that it grows with $q$ (and approaches a constant value at $q \rightarrow \infty$ ).

\section{Worldvolume flux and charge}

The counterpart of (37) for the fixed current case [6] is obtained by setting $q=0$

and retaining a nonzero $J_{0}$ (which is then interpreted as the fixed current). Let us compare the fluxes of $F_{t \rho}$ at $\rho \rightarrow \infty$ for the two cases. They are given by

$$
\begin{aligned}
& F_{t \rho}(\rho \rightarrow \infty)=-\frac{q R^{4}}{\rho^{3}} \quad(\text { fixed } q), \\
& F_{t \rho}(\rho \rightarrow \infty)=-\frac{J_{0}}{\rho^{3}} \quad \text { (fixed current). }
\end{aligned}
$$




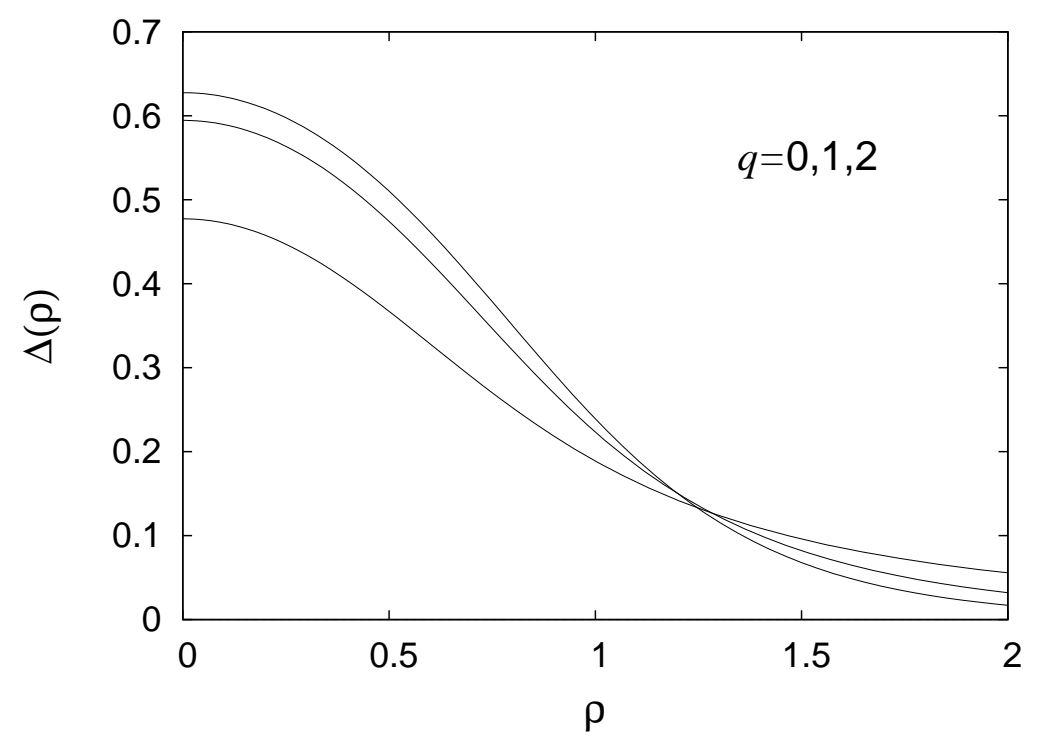

Figure 1: D5 profiles for three values of $q$. Both axes are in units of $R$, and $q$ is in units of $1 / R$. Larger $q$ correspond to larger values of $\Delta(\rho=0)$.

These are obviously similar. Indeed, as we will see shortly, $q R^{4}$ can be interpreted as the average current in the fixed- $q$ case. Note, however, that, unlike (45), which is sourced by charges behind the horizon, the flux (44) is entirely due to charges on the brane.

According to (31), the total charge, coupling to the properly normalized potential $A_{t} /\left(2 \pi \alpha^{\prime}\right)$, is

$$
4 \pi^{3} \alpha^{\prime} \tau_{5} R^{4} \int q d x=N W,
$$

where $W=q L / 2 \pi$ is the winding number, and we have used the expression (16) for $R$ and [17]

$$
\tau_{5}=\frac{1}{(2 \pi)^{5} g_{s}\left(\alpha^{\prime}\right)^{3}}
$$

for the brane tension. Note that (46) is an integer. In the context of the original condensed-matter system, we identify it with the total momentum carried by the current, in units of the Fermi momentum.

Consider a process (a phase slip) in which the winding number $W$ decreases by unity. According to (46), this releases $N$ units of charge from the brane. Since the charge is conserved, some other charged objects must appear. For a different brane arrangement, where a D3 and a D5 have no common spatial dimensions, it is known that a fundamental string stretching between the branes is produced when they cross; 
this is the Hanany-Witten effect [10] in one of its dual versions [11, 12, 13]. Charge conservation leads us to conclude that the same is true here.

To visualize a phase slip, consider the complex position of the $\mathrm{D} 5, \Psi=\Delta e^{i \phi}$, as a function of $\rho, x$, and some interpolation parameter $\tau$ (not necessarily the real time). Define the winding number

$$
W(\tau)=\frac{1}{2 \pi} \int d x \partial_{x} \phi(\tau, x, \rho=0) .
$$

$W$ is a topological invariant (recall that we take the $x$ direction to be a circle): it will stay constant for small fluctuations near a gapped solution. It can change, however, when the D5 passes through the D3s, i.e., $\Delta(\rho=0)=0$ at some values of $x$ and

$\tau$.* The process is shown schematically in Fig. 2, For generality, we consider the case when a zero of $\Delta$ first appears at some nonzero $\rho$ and then propagates to $\rho=0$; alternatively, it may appear at $\rho=0$ directly. When the zero of $\Delta$ reaches $\rho=0$, the D5 crosses the D3s, and light open string modes appear. We expect that this is the point where $N$ open strings required by charge conservation are produced. Since in our case an open string in the D-brane description corresponds to a quasiparticle in the superconductor, we conclude that each phase slip must produce $N$ such quasiparticles. This is precisely the result we have obtained previously by instanton computations on the basis of the Bogoliubov-de Gennes equations [9].

\section{Computation of free energies}

One consequence of quasiparticle production by a phase slip is that it affects the energy balance: the energy gain from unwinding the supercurrent must be weighed against the energy cost of the produced quasiparticles. As a result, when production of quasiparticles is a requirement, phase slips may be energetically forbidden (at zero temperature), and the supercurrent stable. In this section, we describe a numerical computation of the relevant free energies.

Substituting (38) into the expression (33) for the action and integrating by parts in the WZ term, we obtain

$$
S_{D 5}=2 \pi^{2} \tau_{5} \int d t d x\left[-\int d \rho \sqrt{C}\left(1+\Delta_{, \rho}^{2}\right)^{1 / 2}+q R^{4} A_{t}(\infty)\right] .
$$

The integral over $\rho$ here is divergent at $\rho \rightarrow \infty$, but that can be fixed by subtracting some reference $q$-independent expression, e.g.,

$$
\sqrt{C}\left(1+\Delta_{, \rho}^{2}\right)^{1 / 2} \rightarrow \sqrt{C}\left(1+\Delta_{, \rho}^{2}\right)^{1 / 2}-\sqrt{C_{0}},
$$

${ }^{* *}$ In other words, the topological protection is incomplete, as fluctuations of the D5 can "fill in" the space between it and the D3s. Indeed, this is precisely the origin of phase slip processes. 


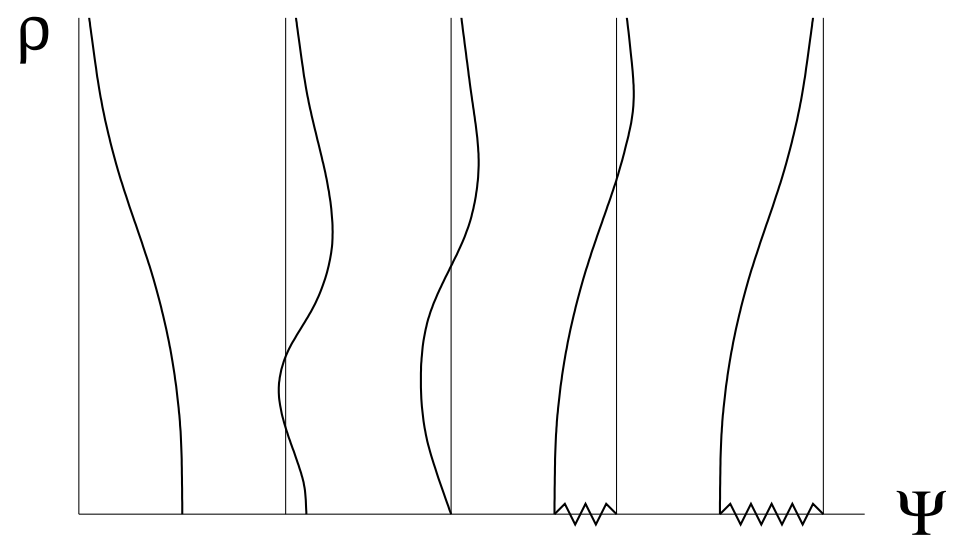

Figure 2: Schematic profiles of some $\Psi(\tau, x, \rho)$ that interpolates between states connected by a phase slip. $\Psi=\Delta e^{i \phi}$ is the complex position of the D5 and is shown here at a fixed $x$ corresponding to the phase-slip center (where it has been chosen real). Curves from left to right correspond to consecutive values of the interpolation parameter $\tau$. They are shifted relative to one another, so that all vertical lines correspond to $\Psi=0$. The D5 crossing the D3s (at $\Psi=\rho=0$ ) corresponds to the profile in the middle. $N$ strings produced at the crossing are shown by a wavy line.

where $C_{0}=\rho^{6}\left(1+R^{4} / \rho^{4}\right)$.

The asymptotic value $A_{t}(\infty)$ appearing in (49) can be interpreted as a chemical potential for the electric current. This can be seen as follows. The average current carried by electrons near the Fermi surface is given by their momentum density in units of $k_{F}$. In the D-brane description, the momentum density is represented by the density of charge conjugate to $A_{t}$. Thus, changing the chemical potential for the current corresponds to a shift

$$
A_{t}(\rho) \rightarrow A_{t}(\rho)+\Lambda_{0},
$$

where $\Lambda_{0}$ is a constant. Allowing the D5 to have a nonzero total charge implies that the physical states of the D5 are only invariant under gauge transformations with parameters vanishing at infinity, in particular, $A_{t}(\rho) \rightarrow A_{t}(\rho)+\Lambda(\rho)$ with $\Lambda(\rho) \rightarrow 0$ at $\rho \rightarrow \infty$. Then, $A_{t}(\infty)$ is a gauge-invariant quantity (a collective coordinate in the terminology of [19]), precisely the one modified by the shift (51)

Variation of (49) with respect to $A_{t}(\infty)$ gives the average current $\bar{I}=2 \pi^{2} \tau_{5} \bar{J}$,

\footnotetext{
${ }^{\dagger \dagger}$ Note that, unlike other identifications of chemical potentials in D-brane systems (for example, the one in [20]), the present argument does not rely on the AdS/CFT dictionary of [3, 4, 5]. That dictionary cannot be used here as $\rho \rightarrow \infty$ corresponds to the asymptotically flat region of the geometry.
} 
where

$$
\bar{J}=q R^{4} .
$$

Legendre transforming the action with respect to $A_{t}(\infty)$ and peeling off $\left(-\int d t\right)$ gives the free energy of the supercurrent state

$$
\mathcal{F}_{\text {super }}(q)=2 \pi^{2} \tau_{5} L \Theta(q)
$$

where

$$
\Theta(q)=\int_{0}^{\infty} d \rho\left[\sqrt{C}\left(1+\Delta_{, \rho}^{2}\right)^{1 / 2}-\sqrt{C_{0}}\right] .
$$

Numerically computed function (54) can be used for a partial analysis of stability of our solutions. In particular, consider classical stability of the solution with respect to "phase separation": formation of a relatively large region along $x$ where $\partial_{x} \phi$ is a constant different from $q$. Classically, formation of such a region should start with a small ( $x$ and $t$-dependent) fluctuation of $\Delta$ and $\phi$ near the original uniform solution. As discussed in Sec. 5, the total winding number can change only when $\Delta$ develops a zero at $\rho=0$. For our solutions, $\Delta(\rho=0)$ is positive, and a small fluctuation will not change that. As a result, phase separation should begin under the condition that the total winding number is unchanged: a decrease of $\partial_{x} \phi$ in a region of $x$ should be compensated by its increase elsewhere. If the region is sufficiently large, its ends give only subleading contributions to the free energy, and the question of stability reduces to that of convexity of $\Theta(q)$. The derivative $d \Theta / d q$ is plotted in Fig. 3. It is monotonically increasing with $q$, which means that the free energy is convex, and phase separation does not occur.

Even if (as the convexity argument suggests) the solution is stable with respect to small fluctuations, it may still be able to decay by large ones - those that allow $W$ to change (phase slips). We now consider various final states to which such a decay might lead.

The first group includes two gapless states: the normal state $\Delta \equiv 0$ and the gapless superconductor found in [6]. A decay to either of these can be visualized as production of a finite density of strings that pull the D5 through the horizon, hiding behind it the worldvolume charge that has originally resided on the brane. The momentum (charge) conservation implies that, to see if either decay is energetically allowed, we must compare the free energy (53) to that of the final state with the same value of the electric flux at infinity. According to (44) and (45), this corresponds to setting $J_{0}$ for either state equal to $\bar{J}$, eq. (52), of the supercurrent state. The free energies of the gapless states are made finite in the same way as (54); thus, for instance, the free energy of the normal state is

$$
\mathcal{F}_{\text {norm }}(q)=2 \pi^{2} \tau_{5} L \int_{0}^{\infty} d \rho\left[\left(C_{0}+J_{0}^{2}\right)^{1 / 2}-\sqrt{C_{0}}\right] .
$$




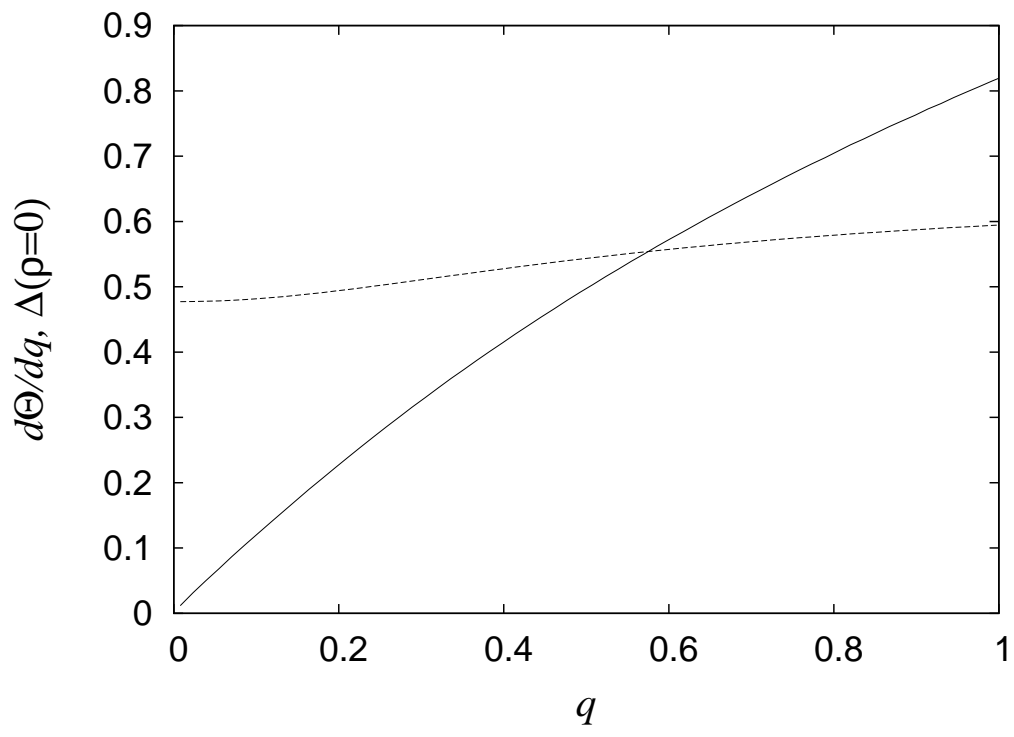

Figure 3: The amount of free energy (in units of $N R / 2 \pi \alpha^{\prime}$; solid line) released by a single phase slip from the supercurrent in a long wire, as compared to the quasiparticle gap (in units of $R / 2 \pi \alpha^{\prime}$; dashed line), both as functions of the winding number density $q$. Intersection of the curves marks the value of $q$ (in units of $1 / R$ ) at which a phase slip becomes energetically allowed. 
Numerically, we find that either of these free energies is always higher than that of the supercurrent state, so the decay is energetically forbidden.

Another type of final state is a gapped supercurrent state with a smaller value of $q$. Here we limit ourselves to states connected by a single phase slip, $\Delta W=-1$, in a wire of macroscopic length $L$. In this case, a phase slip releases approximately $(2 \pi / L) d \mathcal{F}_{\text {super }} / d q$ of free energy from the supercurrent. It, however, requires production of $N$ quasiparticles. The minimal energy of a quasiparticle is given by the quasiparticle gap, $\left(2 \pi \alpha^{\prime}\right)^{-1} \Delta(\rho=0)$. It is convenient to adopt the system of units where all lengths are measured in units of $R$, i.e., $R=1$. In these units, the coefficient in (53) can be written as $2 \pi^{2} \tau_{5}=N / 4 \pi^{2} \alpha^{\prime}$. So, to see if a phase slip is energetically allowed, we need to compare $d \Theta / d q$ to $\Delta(\rho=0)$. The comparison is shown in Fig. 3. We see that the decay becomes possible for $q$ larger than a certain $q_{m}$ (which is somewhat below 0.6 , in units of $1 / R$ ).

\section{Discussion}

Overall, we find that supergravity provides a remarkably detailed picture of a clean (disorder-free) multichannel one-dimensional superconductor. The picture includes the requirement of quasiparticle production by phase slips, seen here as a version of the Hanany-Witten effect (in a non-transverse, non-supersymmetric arrangement of branes). This complements the earlier derivation [9] based on the Bogoliubov-de Gennes equations.

Gapped classical solutions exist in the present case for any winding number density $q$, no matter how large. As we have noted in the introduction, this may be a consequence of momentum conservation, which precludes supplying a nonzero momentum to the superconductor as a whole (the Landau process). It would be interesting to see if gapped solutions seize to exist beyond a certain maximal $q$ in a case when momentum conservation is broken. One such case occurs when the stack of D3s is interrupted by an NS5 brane, in an arrangement similar to those considered in [10]. The numbers of D3s on the two sides of the NS5 need not be equal, so placing a probe D5 in such a geometry will represent a wire with a varying number of transverse channels, i.e., a constriction. The Landau process in this scenario would correspond to formation, at a critical current, of a region near the NS5 where quasiparticles with momenta antiparallel to the flow are gapless. It remains to see, of course, if that is indeed what happens.

Unlike the Landau process, a phase slip does not need to change the total momentum of the system: it can transfer momentum between the supercurrent and quasiparticles. We have seen that beyond a certain $q=q_{m}$ the supercurrent-only 
solution is unstable with respect to decay by phase slips. If we try to set up a supercurrent larger than $J_{m}=q_{m} R^{4}$ in a ring, a part of that current will decay into quasiparticles and, once these form a Fermi surface, a gapless normal component will appear. The latter will exist alongside a superconducting component - as we have seen in Sec. 6, in the present case (a perfectly uniform wire at zero temperature), the normal-only state is never the most energetically favorable.

In the presence of disorder, phase slips can occur without quasiparticle production [9]. Nevertheless, our present results lead us to consider the possibility that, in that case too, a sufficiently large current (now maintained by an external battery) causes appearance of a gapless normal component in the superconducting state. One may contemplate trying to detect such a component experimentally - for instance, by measuring the current-voltage curve of electrons tunneling into the wire off the tip of a scanning tunneling microscope.

This work was supported in part by the U.S. Department of Energy grant DESC0007884.

\section{References}

[1] J. Polchinski, "Dirichlet branes and Ramond-Ramond charges," Phys. Rev. Lett. 75, 4724 (1995) [hep-th/9510017].

[2] G. T. Horowitz and A. Strominger, "Black strings and P-branes," Nucl. Phys. B 360, 197 (1991).

[3] J. M. Maldacena, "The Large N limit of superconformal field theories and supergravity," Adv. Theor. Math. Phys. 2, 231 (1998) hep-th/9711200].

[4] S. S. Gubser, I. R. Klebanov and A. M. Polyakov, "Gauge theory correlators from noncritical string theory," Phys. Lett. B 428, 105 (1998) hep-th/9802109].

[5] E. Witten, "Anti-de Sitter space and holography," Adv. Theor. Math. Phys. 2, 253 (1998) hep-th/9802150.

[6] S. Khlebnikov, "Critical current of a superconducting wire via gauge/gravity duality," arXiv:1201.5103 [hep-th].

[7] W. A. Little, "Decay of persistent currents in small susperconductors," Phys. Rev. 156, 396 (1967).

[8] N. Giordano, "Evidence for macroscopic quantum tunneling in one-dimensional superconductors," Phys. Rev. Lett. 61, 2137 (1988). 
[9] S. Khlebnikov, "Quasiparticle scattering by quantum phase slips in one-dimensional superfluids," Phys. Rev. Lett. 93, 090403 (2004) cond-mat/0311045].

[10] A. Hanany and E. Witten, "Type IIB superstrings, BPS monopoles, and threedimensional gauge dynamics," Nucl. Phys. B 492, 152 (1997) hep-th/9611230.

[11] C. P. Bachas, M. R. Douglas and M. B. Green, "Anomalous creation of branes," JHEP 9707, 002 (1997) hep-th/9705074.

[12] U. Danielsson, G. Ferretti and I. R. Klebanov, "Creation of fundamental strings by crossing D-branes," Phys. Rev. Lett. 79, 1984 (1997) [hep-th/9705084].

[13] O. Bergman, M. R. Gaberdiel and G. Lifschytz, "Branes, orientifolds and the creation of elementary strings," Nucl. Phys. B 509, 194 (1998) [hep-th/9705130].

[14] R. Jackiw and P. Rossi, "Zero modes of the vortex-fermion system," Nucl. Phys. B 190, 681 (1981).

[15] E. J. Weinberg, "Index calculations for the fermion-vortex system," Phys. Rev. D 24, 2669 (1981).

[16] G. 't Hooft, "Symmetry breaking through Bell-Jackiw anomalies," Phys. Rev. Lett. 37 (1976) 8.

[17] J. Polchinski, "String theory. Vol. 2: Superstring theory and beyond," Cambridge, UK: Univ. Pr. (1998) 531 p.

[18] S. R. Coleman, "There are no Goldstone bosons in two-dimensions," Commun. Math. Phys. 31, 259 (1973).

[19] E. Witten, "Bound states of strings and p-branes," Nucl. Phys. B 460, 335 (1996) hep-th/9510135.

[20] R. Apreda, J. Erdmenger, N. Evans and Z. Guralnik, "Strong coupling effective Higgs potential and a first order thermal phase transition from AdS/CFT duality," Phys. Rev. D 71, 126002 (2005) [hep-th/0504151]. 